\title{
Ein Buch mit sieben Siegeln oder ein Segen für ein optimales Krankheitsmanagement?
}

\section{Doris Fischer-Taeschler}

EMBA, Geschäftsführerin Schweizerische DiabetesGesellschaft
Korrespondenz:

Doris Fischer-Taeschler, EMBA Schweizerische Diabetes-

Gesellschaft

Rütistrasse $3 \mathrm{a}$

CH-5400 Baden

Tel. 0562001790

Fax 0562001795

sekretariat@

diabetesgesellschaft.ch
Sind eHealth und Patient ein ungleiches Paar? Sind eHealth und Patient ein Feigenblatt? Die Wahrheit liegt wohl in der Mitte. eHealth kommt, das ist sicher. Die Frage ist: wann und unter welchen Bedingungen? Aber nur, wenn es uns gelingt, das Thema gemeinsam und wirklich zum Nutzen der Patientinnen und Patienten anzugehen und nicht als Feigenblatt für andere Anspruchsgruppen (Ärzte, Krankenkassen, IT-Industrie - die dann den Hauptnutzen für sich beanspruchen wollen).

\section{Dateneinblick für Patienten}

Die Patientinnen und Patienten hätten eigentlich heute schon alles Interesse daran, dass der elektronische Zugang zu den vielen Daten, die über sie gespeichert sind, in einer für sie optimalen Form möglich ist. Und was heisst optimale Form? So aufbereitet, dass der Patient damit auch etwas anfangen kann. Das fängt schon damit an, dass Berichte strukturiert sein müssen, dass sie inhaltlich so formuliert sein müssen, dass auch ein Laie etwas damit anfangen kann, damit sie für den Patienten les- und interpretierbar sind.

Krankenkassen, Spitäler, Ärzte und andere Leistungserbringer haben heute Unmengen von Angaben über ihre Patientinnen und Patienten gespeichert, ohne dass diese davon wissen und ohne dass diese wissen, was mit diesen Daten geschieht. Bedauerlich ist allerdings vor allem, dass sie diese Informationen nicht für sich nutzen können, und dass dies alles ohne ihr explizites Einverständnis geschieht. Da werden Bytes um Bytes gesammelt, unstrukturiert und damit auch nicht formell zugänglich, und der Patient hat keine Ahnung davon. Er weiss nicht, ob die Krankenkasse damit Risikoselektion betreibt, er weiss nicht, welche Daten unter welchem Stichwort von seinem Dossier beim Arzt ins Dossier des Spitals wechseln und vice versa. Er gibt sein Rezept in der Apotheke ab, erhält die verschriebenen Medikamente, weiss aber nicht, was alles nachher in elektronischer Form über dieses Rezept registriert und ausgetauscht wird.

Dies müsste aus Patientensicht zu einer Ablehnung dieser neuen Technologien führen. Aber: aus Sicht der Patienten gibt es keine Wahl - der technologische Fortschritt findet ohne unsere ausdrückliche Zustimmung statt - eine Vogel-Strauss-Politik wäre fatal. Wir müssen Ja sagen zum Fortschritt. Ja sagen $\mathrm{zu}$ den technischen Neuerungen, die unweigerlich auf uns zukommen. Wir lernen von Jahr zu Jahr die modernen elektronischen Hilfsmittel besser kennen und nutzen. Die neuen Technologien, insbesondere die Möglichkeit, jederzeit online auf jedwede elektronische Information zurückgreifen $\mathrm{zu}$ können manchmal auch zu nicht optimalen Bedingungen, im Zug oder an einer Sitzung, haben rasch Fuss gefasst, und viele von uns sind damit vertraut geworden. Weshalb diesen nützlichen Fortschritt nicht für den Alltag nutzen?

\section{Hilfreiche eHealth-Plattformen}

Es gibt auch bereits einige funktionierende Beispiele von eHealth-Plattformen. Gerade für Diabetikerinnen und Diabetiker ist die Web-Applikation von «Evita» positiv hervorzuheben, weil sie nicht einfach ein zusätzliches Tool ist, sondern Betroffenen hilft, für ein gutes Diabetesmanagement wichtige Informationen zentral zu speichern. Ebenso kennen einige Krankenkassen bereits eigene webbasierte Plattformen, die ihren Mitgliedern echten Mehrwert bieten.

\section{Wir müssen Ja sagen zum Fortschritt.}

Wichtig scheint uns, dass es Applikationen sind, die von allen Akteuren, Leistungserbringern und Patientinnen und Patienten gemeinsam und zugleich einfach genutzt werden können. Die Akzeptanz für diese Instrumente wird denn auch in ihrer Einfachheit und Benutzerfreundlichkeit liegen.

Wir plädieren dafür, dass die bereits existierenden elektronischen Plattformen (z.B elektronische Krankengeschichte) in die neuen Applikationen einbezogen werden. Kein Arzt wird zusätzliche Zeit investieren, um ausserhalb seiner ihm bekannten Software noch anderswo elektronische Daten zu hinterlegen oder gar zu erfassen. Und auch Patientinnen und Patienten wollen nicht noch einmal von Hand erfassen, was ein Gerät bereits getan hat. Es geht also letztendlich darum, die Schnittstellen, die Formate und die formalisierten Inhalte zu definieren, um diese dann aus heutigen Standardapplikationen 
direkt versorgen zu können. Erst dann werden die vielgerühmten Synergieeffekte auch wirklich wirksam werden.

\section{Wer hat die Datenhoheit?}

Selbstverständlich gilt es, die Zugriffsrechte zu regeln. Damit steht und fällt der Datenschutz. Wir fordern, dass die Datenhoheit bei der Patientin, dem Patienten sein muss, allenfalls bei einer von ihm bezeichneten Vertrauensperson. Diese Garantie - dass er immer die Datenhoheit hat - scheint uns die Grundvoraussetzung zu sein, damit das System auch langfristig funktionieren wird. Die Patientinnen und Patienten werden das dazu notwendige Vertrauen nur haben, wenn der letzte Entscheid bei ihnen liegt. Zur Not müssen wir auch akzeptieren, dass diejenigen, die die Datenhoheit haben, auch einmal einen Entscheid fällen, der sich negativ auswirken kann. Damit das nicht geschieht, braucht es Information und Schulung - und zwar bereits zu einer Zeit, an dem sie erst potentielle Patientinnen und Patienten sind und unser Gesundheitswesen noch nicht übermässig nutzen (müssen). Sie müssen wissen, was eine Freigabe in der elektronischen Patientenakte für sie bedeuten kann - im Positiven wie Negativen.

\section{Wir fordern, dass die Datenhoheit bei den Patientinnen und Patien- ten sein muss.}

Als Patientenorganisation sind wir aber auch gefordert, kritisch zu sein. Ich möchte nicht falsch verstanden werden: Es geht weder darum, alles unter dem Stichwort «Datenschutz» und «Schutz des Patienten» abzulehnen, noch darum, alles euphorisch zu unterstützen. Mit kritisch Betrachten meinen wir vor allem das Hinterfragen des Nutzens für die Patientinnen und Patienten. Es muss für sie z.B. einen Mehrwert an Information (nützlich und verständlich), einen Mehrwert in der Qualität der Behandlung (schneller gesund) oder einen Mehrwert geben in Form von nicht doppelt gemachten Untersuchungen (= weniger Kosten und weniger Interventionen z.B beim Röntgen, im Labor usw.). Erst dann werden die Patientinnen und Patienten nicht mehr als Feigenblatt für Ansprüche gewisser anderer Gruppen dienen. Und diesen Zustand wünschen wir uns.

\section{InfoSocietyDays Bern: Swiss eHealth Forum} Im Rahmen der InfoSocietyDays in Bern findet am 10. und 11. März das Swiss eHealth Forum statt. Die diesjährigen Leitthemen sind:

Donnerstag, 10. März 2011

Consumer Driven eHealth - Der Einfluss der Gesellschaft auf die Entwicklung von eHealth

Freitag, 11. März 2011

Integrierte Gesundheitsversorgung - Welchen Beitrag leistet eHealth?

Vertreter aus Gesundheitswesen, Wissenschaft, Wirtschaft und Politik beleuchten das Thema eHealth in Plenumsreferaten. In Solution-Präsentationen legen Anbieter, Problemlöser und Anwender Erfahrungsberichte vor, sprechen über Erwartungen, Hemmnisse und realisierten Nutzen. Der zweite Forumstag bietet einen speziellen IHE Thementrack.

Das Swiss eHealth Forum ist Teil der InfoSocietyDays 2011, die vom 7. bis 11. März durchgeführt werden. Mit den drei Foren «eEconomy», «eGovernment» und «eHealth» behandeln die InfoSocietyDays als einzige Schweizer Plattform drei wichtige Kernthemen unserer Informationsgesellschaft. Das detaillierte Forumsprogramm, weitere Informationen sowie die Möglichkeit, sich anzumelden, finden Sie unter www.infosocietydays.ch

Die Schweizerische Ärztezeitung ist Medienpartner der InfoSocietyDays. 